\title{
Theoretical and practical aspects of the Coandă effect applied in aeronautics
}

\author{
Ionică Cîrciu ${ }^{1 *}$, and Constantin Rotaru ${ }^{1}$ \\ ${ }^{1}$ Department of Aviation, "Henri Coandă" Air Force Academy, 160 Mihai Viteazul Street, 500183 \\ Braşov, Romania
}

\begin{abstract}
The Coandă Effect, or better said, the deviation of fluid jets to stay attached to a convex surface is a complex gas-dynamic effect, named after the Romanian aviation engineer Henri Coandă and it was for the first time highlighted by him in 1910 during the take-off of one of his first planes, Coandă-1910, which was also the first jet plane to fly. During the short flight, Coandă was able to notice the near-fit alignment of the flue gas jets with the airplane fuselage. Later, both Coandă and other scholars have intensively studied the effect that is named The Coandă Effect, in his honor.
\end{abstract}

\section{Introduction}

The purpose of this paper is to point out that the use of the Coanda effect in the propulsive field is still a topical subject, because despite the numerous theoretical and experimental analyzes conducted over the years, we can not say that a definitive conclusion on capabilities, the possibilities of application and the performance of devices using the Coanda effect. We consider a two-dimensional jet-attached flow.

Translatation results in a $3 \mathrm{D}$ rectangular ejection device, which can also work with a limiting wall, see fig. 1 .
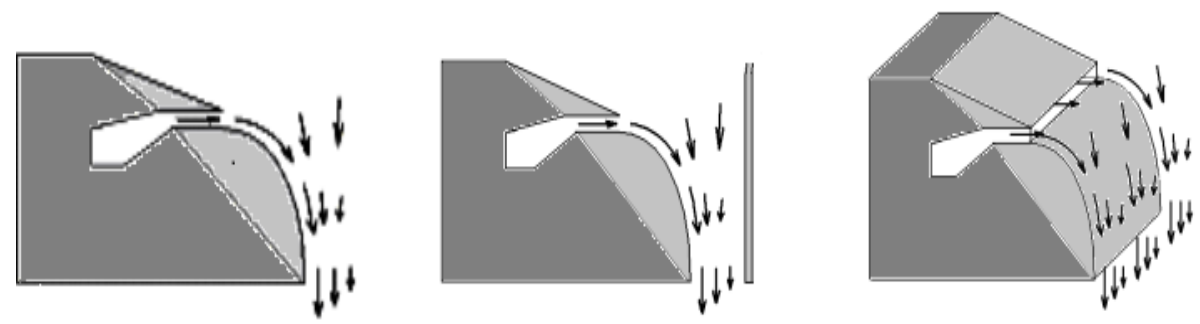

Fig.1. Ejection device: a) Coanda 2D flow; b) Rectangular ejection device; c) 3D rectangular ejection device with limitation wall

* Corresponding author: circiuionica@yahoo.co.uk 


\section{Theoretical aspects of the Coandă effect}

In figure 2, section 0-0 is that of the opening, through which the primary flow, driven by compression, acceleration or isentropic drive, and direct aspiration from the environment is introduced. The h-h section is the suction section through which only the flow is drawn and is characterized in that the total flow enthalpy is even that of the $i_{H^{*}}$ medium. The area of point $A$ is supposed to represent the point of maximum depression of the flow. Section B-B is the end of the profile (line $0 \mathrm{AB}$ ). Section ,'C $-\mathrm{C}$ "' is the final section of the aspiration, characterized in that the size of the mixing range is the same as that of the passage section 'C - C'. Section 'D-D' is the exhaust section of the Coanda device, characterized in that the static pressure is equal to the static pressure of the $\mathrm{p}_{H}$ environment.

The ," h-0-C-B-h" domain is the aspiration domain, characterized in that the total enthalpy of the flow is even that of the $i_{H^{*}}$ medium. The ,' 0 -ABC-C- 0 " domain is considered to be the domain receiving the entire amount of flow trained through the permeable surface C0. The C-D-D-C domain is considered to be the domain of alignment of the gas-dynamic parameters in section $\mathrm{C}-\mathrm{C}$, usually having a divergent shape to improve the efficiency of the Coanda device [3-4].

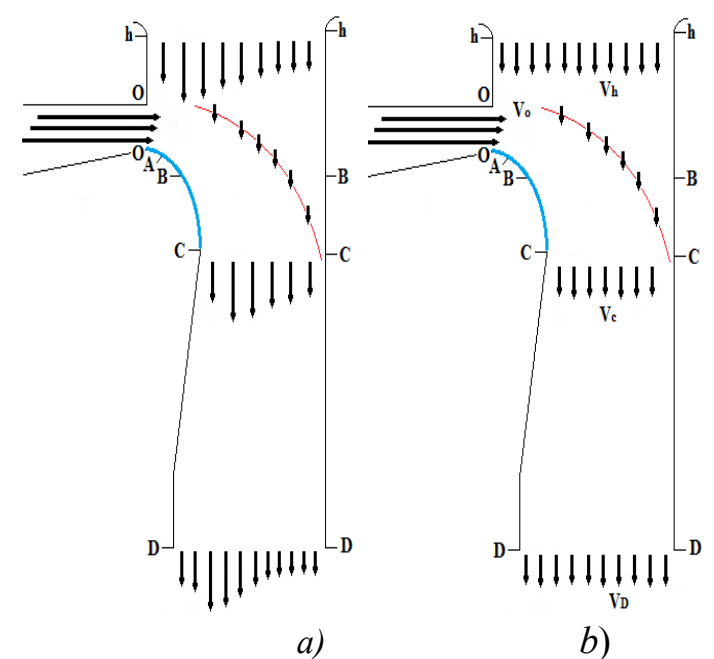

Fig. 2. Coandă device: a) non uniform speed repartition, b) uniform speed repartition

This theoretical analysis will be considered as a fixed motion for an isentropic, compressible flow, minimizing the influence of friction forces, thermal transfer from the wall, the effects of the boundary layer between the jet and the Coanda profile. Calculation formulas to be used for the braked enthalpy $i^{*}$ at a point of a section $\mathrm{S}$, for the mass flow $\mathrm{M}$, the propulsion force $\mathrm{F}$ of a propeller (field of analysis) having the exhaust section considered, the absolute power $\mathrm{P}$ present in the fluid in a certain flow section and the power introduced into the fluid $\mathrm{P}_{\mathrm{f}}$ are:

$$
i^{*}=i_{H}^{*}\left(\frac{p}{p_{H}}\right)^{\frac{k-1}{k}}+\frac{V^{2}}{2}
$$




$$
\begin{gathered}
M=\int_{\mathrm{S}}^{*} \rho \mathrm{VdS} \\
\mathrm{F}=\int_{\mathrm{S}}\left[\rho \mathrm{V}^{2}+\left(\mathrm{p}-\mathrm{p}_{\mathrm{H}}\right)\right] \mathrm{dS}
\end{gathered}
$$

We remind you of environmental conditions for static pressure $\mathrm{p}_{\mathrm{H}}$, density $\rho$, the jet speed $\mathrm{V}$ and braked enthalpy $\mathrm{i}_{\mathrm{H}}{ }^{*}$.

$$
P_{f l}=\int_{S} \rho V\left(i^{*}{ }^{i}{ }^{*} H\right) d S
$$

to which is added the expression of density variation according to static pressure, for isentropic transformation

$$
\rho=\rho_{\mathrm{H}}\left(\frac{\mathrm{p}}{\mathrm{p}_{\mathrm{H}}}\right)^{\frac{1}{k}}
$$

We consider a propeller that puts the fluid in motion by an isentropic mechanical transfer (propeller, blower, jet, etc.) at a fixed point and having a complete expansion in the $\mathrm{S}_{\mathrm{ev}}$ discharge section through which the $\mathrm{V}_{e v}$ velocity (uniform in section) flows mass flow $\mathrm{M}_{\mathrm{ev}}$, the transferred (introduced) power to the fluid being $\mathrm{P}_{f}$. It is possible to write the expression of the flow: the reaction force $\mathrm{F}$ (traction) and the power introduced into the Pfl fluid as shown below:

$$
\begin{aligned}
& M_{e v}^{*}=\left(\rho_{H} V_{e v} S_{e v}\right) \text { have } \mathrm{F}=M_{e v}^{*} \mathrm{~V}_{\mathrm{ev}}=\left(\rho_{H} V_{e v}^{2} S_{e v}\right) \\
& \text { then: } \mathrm{P}_{\mathrm{fl}}=M_{e v}^{*} \mathrm{~V}_{\mathrm{ev}} / 2=\left(\rho_{H} \frac{V_{e v}^{3}}{2} S_{e v}\right)
\end{aligned}
$$

Under these conditions, the power transmitted to the $\mathrm{P}_{\mathrm{fl}}$ fluid up to the $\mathrm{S}_{\mathrm{ev}}$ section can be correlated as follows: speed - section; speed-flow; speed-force $[4,8]$.

$$
\begin{aligned}
& P_{f l}=\eta P_{0}=\left(\rho_{H} \frac{V_{e v}^{3}}{2} S_{e v}\right)=M_{e v}^{*} \frac{V_{e v}^{2}}{2}=\mathrm{F} \frac{V_{e v}}{2} \\
& \text { then }: \mathrm{F}=\frac{2 P_{f l}}{V_{e v}}=2 \frac{\eta \mathrm{P}_{0}}{\mathrm{~V}_{\mathrm{ev}}}
\end{aligned}
$$

\section{Propulsion efficiency}

We will call the $E_{\text {f- }}$ efficiency of a propulsion device as the ratio of the power generated (generated) by the device and the power it consumes usually having the unit of measure $\mathrm{kg}$ /hp. We can now point out that a propeller can have increased efficiency even if it has a low efficiency (Table 1). For example for $=20 \%$ and $\mathrm{V}_{\mathrm{ev}}=20 \mathrm{~m} / \mathrm{s}$, the efficiency reaches a value of $0.020 \mathrm{~N} / \mathrm{W}$, i.e. about $1.489 \mathrm{~kg} / \mathrm{h}$ where we would have a propeller with $\mathrm{n}=80 \%$ and $\mathrm{V}_{\mathrm{i}}=160 \mathrm{~m} / \mathrm{s}$ and identical to a propeller with $\mathrm{n}=80 \%$ and $\mathrm{V}_{\mathrm{i}}=80 \mathrm{~m} / \mathrm{s}$. This leads to the conclusion that there may be some cases where, in order to achieve high efficiency, it is 
preferable to design, to abandon a high energy transfer efficiency, which is important to have a / increased ratio. Thus, the force produced by a propeller can increase, although the energy consumed remains the same.

Table 1. Effectiveness Ef values reported at yield and uniform section speed

\begin{tabular}{|c|c|c|}
\hline$\eta$ & $V_{e v}$ & $E_{f}=\frac{F}{P_{0}}=\frac{2 \eta}{V_{e v}}$ \\
\hline $20 \%$ & $20 \mathrm{~m} / \mathrm{s}$ & $1,489 \mathrm{~kg} / \mathrm{CP}$ \\
\hline $80 \%$ & $80 \mathrm{~m} / \mathrm{s}$ & $1,488 \mathrm{~kg} / \mathrm{CP}$ \\
\hline $80 \%$ & $160 \mathrm{~m} / \mathrm{s}$ & $0,744 \mathrm{~kg} / \mathrm{CP}$ \\
\hline
\end{tabular}

We consider a Coanda uniform ejection device considered to be a reference, since in reality there can not be such a case. We take two analytical sections, 0-0 and D-D, we obtain:

$$
\begin{aligned}
& \mathrm{P}_{0}=\rho_{H} \frac{V_{0}^{3}}{2} S_{0}=M_{0} \frac{V_{0}^{2}}{2}=\mathrm{F}_{0} \frac{V_{0}}{2} \\
& \mathrm{P}_{\mathrm{D}}=\eta_{D} \mathrm{P}_{0}=\rho_{H} \frac{V_{D}^{3}}{2} S_{D}=M_{D} \frac{V_{D}^{2}}{2}=\mathrm{F}_{\mathrm{D}} \frac{\mathrm{V}_{\mathrm{D}}}{2}
\end{aligned}
$$

\section{Modeling and simulation of the Coanda effect}

Coandă Effect Small helicopter is an experimental validation of using Coanda effect on the tailboom. A helicopter structure consists of two main components: a cabin and a tailboom of materials composite (fiberglass and carbon fibre).

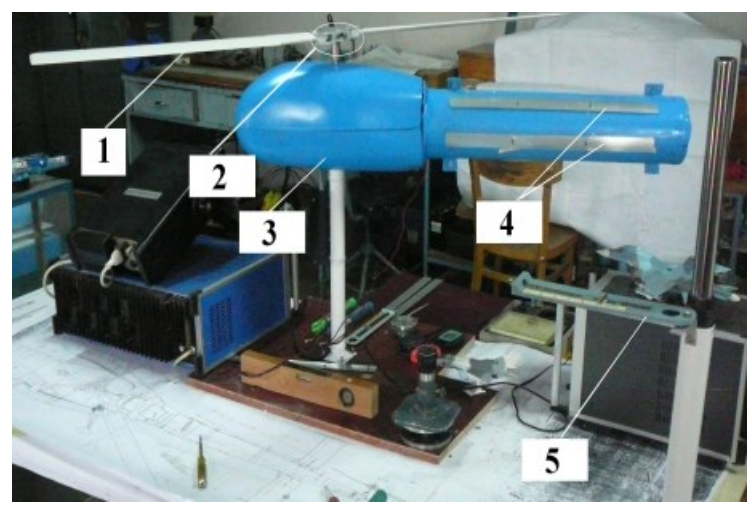

Fig. 3. Experimental device: 1- carbon fibre helicopter blades, 2-hub pitch, 3- fiberglass structure, 4Coandă slots, 5 - the force measuring device

In the Fig.3 the pitch of the hub and the carbon fiber blades can be seen. This experiment aims to highlight the effect of Coanda and to indicate propulsion performance. For this purpose, beam characteristics have been gradually modified, trying to obtain a stronger- $\mathrm{F}$ force. The experimental model will show the flow of fluid along the tail. [4] 
This was simulated on a Coanda optimally profile adjustable, depending on the flows data load-bearing rotor, the intubated fan from the tailboom, and the location and geometry of the slot. A detail element on the constructive shape of the Coanda slit can be distinguished in fig.4 . It is possible to observe the flow of Coanda (blue color) that is the area of the boundary layer that will become Coanda's profile.
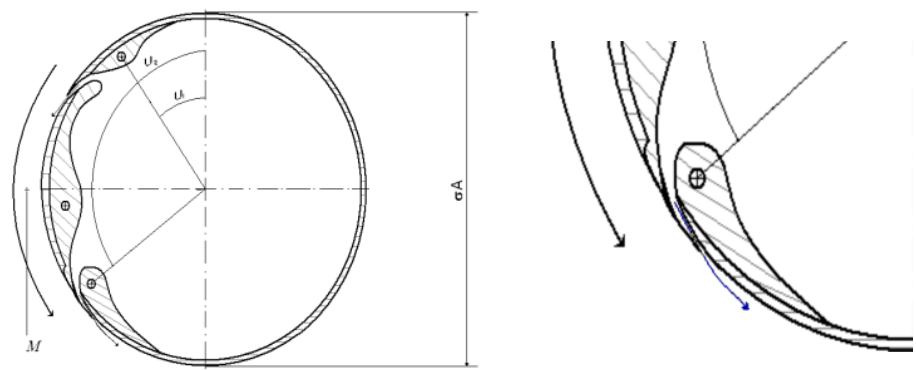

Fig.4. Detail elements of the Coanda profile

This makes us have a new favorable arrangement for creating more convenient lateral forces. more powerful, low energy consumption compared to those used in basic relationships describing helicopter efficiency. [6]

The geometric elements of the Coanda slit to achieve a flow without disturbance will require practical adjustments on the experimental device to determine optimal shapes, the proprietary model proposed in Figure 5 will be subjected to a computer simulation analysis.

The interest is to compute the flow particularities around some aerodynamic shapes with a particular focus on the tail of Aluette helicopter.

The results are presented in Fig. 5 .
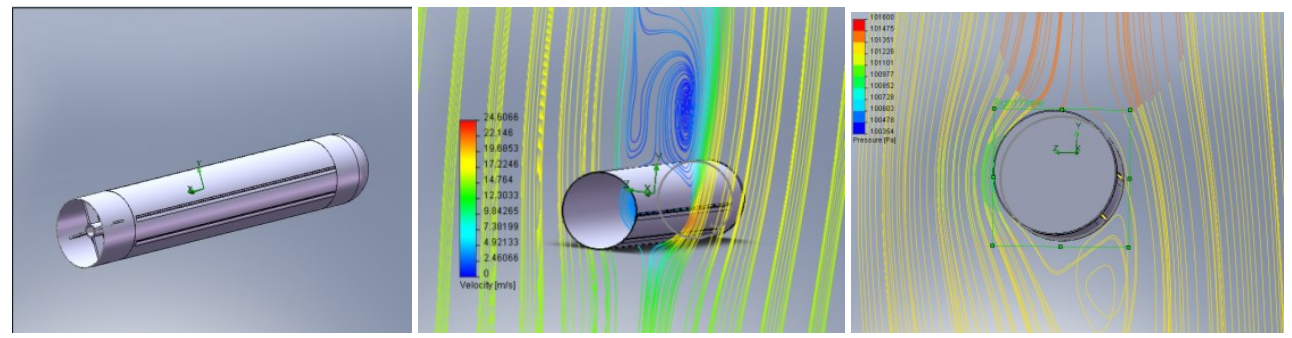

Fig. 5. Simulation of the flow around the tail helicopter

The numerical simulations were made with a Fluent 6.1 software program that allows modeling of the flows around a body.
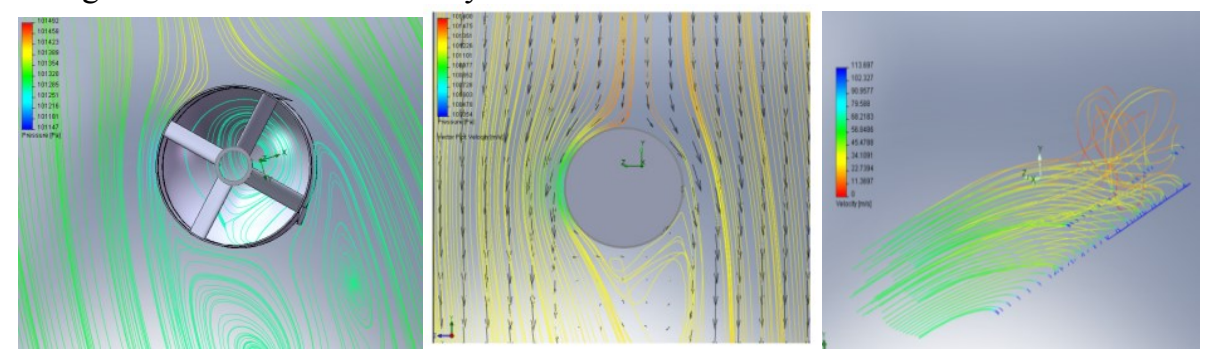

Fig. 6. A full 3D view of the stream around the tail 
In Fig.6, the increase in the speed of the current lines both inside and outside the queue beam results in the occurrence of the Coandă effect seen in the color code by making a depression in the slot area. We note that the current lines drawn by the bearing rotor form on the slit side a depression area which results in a lateral force $F$ due to the Coanda effect.

Tables 2 and 3 show the values of the lateral force due to the Coandă effect at a vertical speed $V_{r}$ of the power lines of $18 \mathrm{~m} / \mathrm{s}$ and $V_{r}=12 \mathrm{~m} / \mathrm{s}$ respectively, depending on the variation of the speed of the flow within the tail boom. Ten values have been selected for interpretation and graphic presentation of force $\mathrm{F}$ according to speed $\mathrm{V}_{\mathrm{c}}[6]$.

Table 2 shows the values of lateral force $F$ depending on the variation of the speed of the fluid through the tail boom $\mathrm{V}_{\mathrm{c}}$ by maintaining the vertical air current at a constant speed $\mathrm{V}_{\mathrm{r}}=18 \mathrm{~m} / \mathrm{s}$.

Table 2. Vertical speed $\mathrm{V}_{\mathrm{r}}$ of the power lines of $18 \mathrm{~m} / \mathrm{s}$

\begin{tabular}{|c|c|c|c|c|c|}
\hline $\begin{array}{c}\text { Nr. } \\
\text { crt }\end{array}$ & $\begin{array}{c}\text { Static } \\
\text { pressure } \\
{\left[\mathrm{N} / \mathrm{m}^{2}\right]}\end{array}$ & $\mathrm{Vc}[\mathrm{m} / \mathrm{s}]$ & $\begin{array}{c}\text { Force } \\
\mathrm{F}[\mathrm{N}]\end{array}$ & $\begin{array}{c}\text { Fy } \\
{[\mathrm{Nm}]}\end{array}$ & $\begin{array}{c}\mathrm{Fz} \\
{[\mathrm{Nm}]}\end{array}$ \\
\hline 1 & 101237,46 & 4,190 & 2,291 & 0,0637 & $-0,0101$ \\
\hline 2 & 101239,15 & 4,193 & 2,312 & 0,0681 & $-0,0121$ \\
\hline 3 & 101240,74 & 4,21 & 2,407 & 0,0726 & $-0,0145$ \\
\hline 4 & 101242,47 & 4,29 & 2,518 & 0,0768 & $-0,0165$ \\
\hline 5 & 101244,16 & 4,34 & 2,627 & 0,0809 & $-0,0182$ \\
\hline 6 & 101245,99 & 4,38 & 2,701 & 0,0859 & $-0,0184$ \\
\hline
\end{tabular}

$\mathrm{Vc}$ - the speed of the fluid through the tail boom;

$\mathrm{F}$ - lateral force resulted from the Coandă effect,

Fy- torque on direction y, Fz-torque on direction $\mathrm{z}$.

Table 3 shows the values of lateral force $F$ depending on the variation of the speed of the fluid through the tail boom $\mathrm{V}_{\mathrm{C}}$ by maintaining the vertical air current at a constant speed $\mathrm{V}_{\mathrm{R}}=12 \mathrm{~m} / \mathrm{s}$.

Table 3. Vertical speed $V_{\mathrm{r}}$ of the power lines of $12 \mathrm{~m} / \mathrm{s}$

\begin{tabular}{|c|c|c|c|c|c|}
\hline $\begin{array}{c}\text { Nr. } \\
\text { crt }\end{array}$ & $\begin{array}{c}\text { Static } \\
\text { pressure } \\
{\left[\mathrm{N} / \mathrm{m}^{2}\right]}\end{array}$ & $\mathrm{Vc}[\mathrm{m} / \mathrm{s}]$ & $\begin{array}{c}\text { Force } \\
\mathrm{F}[\mathrm{N}]\end{array}$ & $\begin{array}{c}\text { Fy } \\
{[\mathrm{Nm}]}\end{array}$ & $\begin{array}{c}\mathrm{Fz} \\
{[\mathrm{Nm}]}\end{array}$ \\
\hline 1 & 101229,63 & 4,122 & 2,00683 & 0,03137 & $-0,00261$ \\
\hline 2 & 101229,78 & 4,178 & 2,027 & 0,03347 & $-0,002413$ \\
\hline 3 & 101229,98 & 4,210 & 2,076 & 0,035 & $-0,00089$ \\
\hline 4 & 101230,07 & 4,290 & 2,103 & 0,037 & 0,00080 \\
\hline 5 & 101230,32 & 4,340 & 2,131 & 0,039 & 0,0009 \\
\hline 6 & 101230,72 & 4,386 & 2,153 & 0,041 & 0,0019 \\
\hline
\end{tabular}

The diagram in Fig. 7 shows the increase of lateral force F due to the Coandă effect and depending on speed $\mathrm{V}_{\mathrm{c}}$, through rounding the values to two decimal digits.

There is a linear increase of force F, on small areas, compared to the mild increase of the speeds of the air stream through the tail boom, which makes us conclude that we can reach an increase of the lateral force $\mathrm{F}$ by mild variation of the rotation speed of the fan. 


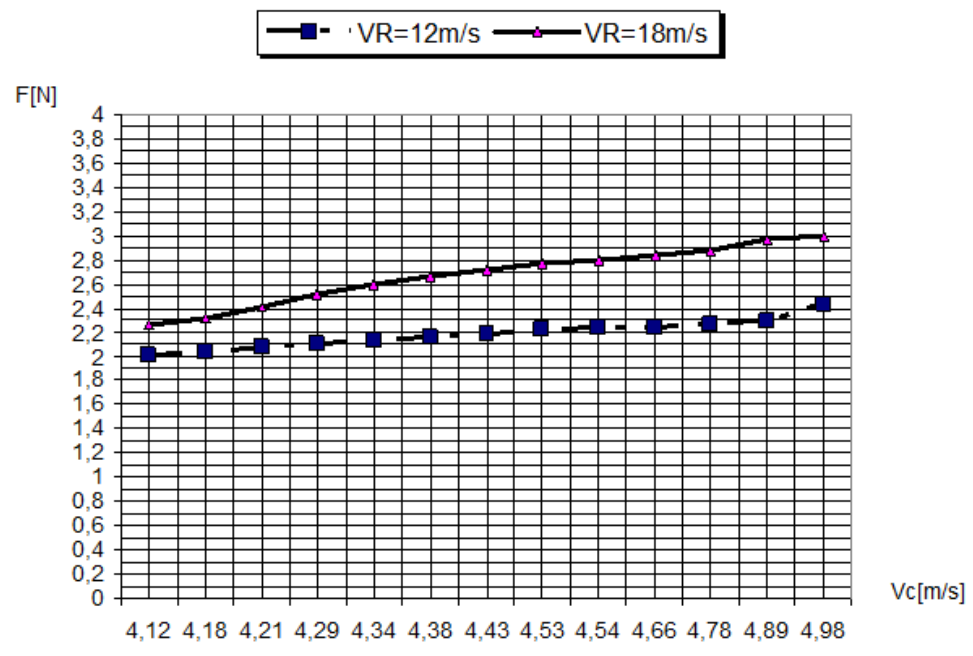

Fig. 7. A comparative analysis of the variation of the lateral force for a constant $V_{R}$ of $18 \mathrm{~m} / \mathrm{s}$ and 12 $\mathrm{m} / \mathrm{s}$ respectively.

\section{Conclusions}

- There may be some cases where, in order to achieve high efficiency, it is preferable to design, to abandon a high energy transfer $\eta$ efficiency, it is important to have a report $\eta / \mathrm{V}_{\mathrm{ev}}$ grown. Thus, the force produced by a propulsion device may increase, although the power consumed remains the same.

- It has been found practically that obtaining a convenient force due to the Coandă effect, which can be easily modified, requires the arrangement of directional adjustable elements along the length of the slots.

- The geometric and input parameters were correctly chosen, and the experimental results were quite similar to those simulated by the computer;

- In order to obtain as much force as possible for available energy, it is preferable to seek to move as much fluid as possible at a lower speed instead of a small amount of high -velocity fluid.

\section{References}

1. H. Coandă,"'Air - source du progrès"'[Book], 1961.

2. I. Cîrciu, S. Dinea,. Review of applications on Coandã effect. History, theories, new trends, Review of the Air Force Academy, 2(17) (2010)

3. V. Drăgan. Preliminary aerodynamic evaluation of a supersonic stealth intake. Review of the Air Force Academy, Brasov, 9(2), 29-32 (2011)

4. S. Cîrciu, S. Dinea, M. Boşcoianu. Review of contributions regarding Coanda effect, Review of the Air Force Academy, 2 (5)2009.

5. I. Cîrciu, M. Boşcoianu. An analysis of the efficiency of Coandă-NOTAR anti-torque systems for small helicopters, INCAS Bulletin, 2(4), (online) ISSN 2247-4528, (print) ISSN 20668201, ISSN-L 2066-8201, DOI: 10.13111/2066-8201.2010.2.4.12, pp.81-88 (2010) 
6. I. Cîrciu, D. Luculescu, V. Prisacariu, E.M.C. Rotaru. Theoretical Analysis and Experimental Researches regarding the Asymmetrical Fluid Flow Applied in Aeronautics, Advances in Materials Science and Engineering, ID 681284, doi:10.1155/2015/681284 (2015)

7. C. Berbente, S. Dinea. On Kármán model for Coanda ejector with incompressible flow, Scientific Bulletin of University „Politehnica”' of Bucharest 71(2), 19 (2009)

8. V. Drăgan.Teorii Asupra Efectului Coandă (ISBN 978-973-0-13821-4, Bucuresti 2012) 